\title{
Contribución al análisis del impacto de la creación de una red de vías ciclistas en la economía local y en la imagen turística de las ciudades. El caso de Sevilla
}

\author{
Contribution to the analysis of the impact of the creation of a \\ network of cycle-paths in the local economy and in the touristic \\ image of cities. The case of Seville
}

\section{Ricardo Marqués, ${ }^{1}$ Manuel López-Peña ${ }^{2}$ y Vicente Hernández-Herrador ${ }^{3}$}

Fecha de recepción: 02-03-2020 - Fecha de aceptación: 16-06-2020

Hábitat y Sociedad (ISSN 2173-125X), n. ${ }^{\circ}$ 13, noviembre de 2020, pp. 193-206.

http://dx.doi.org/10.12795/HabitatySociedad.2020.i13.11

\section{Summary}

This paper analyzes the impact of the creation of a continuous network of segregated cycle paths on local economy and on the touristic image of cities, through the case study of the city of Seville by developing a methodology that could be applied to the analysis of other similar experiences. The implementation of a network of bike tracks in the city from 2007 onwards led to the revitalization of a sector, quite marginal until that date, turning it into a dynamic and growing sector in an environment of economic crisis, with high job creation rates per euro billed, something quite beneficial in a city with high rates of unemployment. The impact of the creation of the mentioned network of cycle paths in the touristic image of the city, something very important given the relevance of the tourism in its economy, is apparent from the inclusion of the aforementioned infrastructure in the main international tourist guides of the city. The microanalysis of the opinions of the visitors, users of said infrastructure, evidences a positive valuation, and its high multiplier power as an argument for recommending Seville as a tourist destination in their immediate social environments. However, some deficiencies that should still be corrected are detected, such as conservation problems, or the low level of knowledge of such infrastructure at the international level.

\section{Key words}

Sacycle paths; Bicycle economy; Sustainable tourism; Local economy

\section{Resumen}

Este trabajo analiza el impacto de la creación de una red continua de vías ciclistas segregadas del tráfico motorizado en la economía local y en la imagen turística de las ciudades, a través del estudio de caso de la ciudad de Sevilla, desarrollando para ello una metodología que podría ser útil en el análisis de otras experiencias similares. La implantación de una red de vías ciclistas en la ciudad a partir de 2007 supuso la dinamización de una actividad económica hasta esa fecha bastante marginal, convirtiéndola en un sector dinámico y en crecimiento en un entorno de crisis económica, con unas ratios de creación de empleo por euro facturado muy interesantes en una ciudad azotada por un paro endémico. El impacto de la creación de vías ciclistas en la imagen turística de la ciudad, algo muy importante dada la relevancia del sector turístico, se hizo evidente desde el principio a partir de la inclusión de dicha infraestructura en las principales guías turísticas internacionales. El microanálisis de las impresiones de los visitantes, usuarios de dicha infraestructura, evidencia su alta valoración y su alto poder multiplicador como argumento a la hora de recomendar Sevilla como destino turístico en su entorno social inmediato. No obstante, se detectaron algunas deficiencias que necesitan corregirse, como problemas de conservación y el escaso conocimiento de la infraestructura a escala internacional.

\section{Palabras clave}

Vías ciclistas; Economía de la bicicleta; Turismo sostenible; Economía local

1 Catedrático en la Facultad de Física de la Universidad de Sevilla. Responsable del Servicio Integral de la Bicicleta de la Universi-

dad de Sevilla (SIBUS) y presidente de la asamblea ciclista A Contramano. E-mail: marques@us.es. ORCID: 0000-0003-0528-384X

2 Ingeniero de organización de empresas y consultor independiente. E-mail: mlp_vega@hotmail.com

3 Consultor independiente. E-mail: vicenth_arq@hotmail.com. ORCID: 0000-0003-2223-8223 


\section{Introducción}

El creciente reconocimiento de la importancia del fomento de la movilidad sostenible y la salud pública ha disparado el interés hacia las políticas de fomento de la bicicleta como un modo de transporte a la vez sostenible y saludable. La bicicleta puede jugar un papel importante en la movilidad urbana para distancias del orden o inferiores a los $5 \mathrm{~km}$, además de garantizar el acceso intermodal al transporte público en áreas de baja densidad de población (Dekoster y Scholaert, 1990; Sanz, 1996; Marqués, 2017), contribuyendo de ese modo a enmendar la insostenibilidad del actual modelo de transporte en España (Estevan y Sanz, 1996; Sanz, Vega y Mateos, 2014) y en Andalucía (Sanz et al., 2015). Asimismo, la bicicleta usada como modo de transporte cotidiano puede jugar un importante papel en la prevención de muchas dolencias ligadas a los modos de vida sedentarios, provocados en parte por el actual modelo de movilidad, como las enfermedades coronarias o la obesidad, lo que la convierte en una importante aliada de las políticas de salud pública (WHO, 2000).

La ciudad de Sevilla no ha sido ajena a esta corriente internacional de creciente apoyo hacia el ciclismo urbano. Así, desde el año 2006 se están llevando a cabo en la ciudad políticas e iniciativas para el fomento de la bicicleta con notable éxito (Marqués, Hernandez-Herrador, Calvo-Salazar y García-Cebrian, 2015) en el marco del Plan de la Bicicleta 2007-2010 (Ayuntamiento de Sevilla, 2007) primero y del Programa de la Bicicleta 2014-2020 (Ayuntamiento de Sevilla, 2014) con posterioridad.

El análisis de los efectos económico de tales políticas es uno de los factores a considerar a la hora de juzgar la validez y las consecuencias de las mismas, así como para planificar futuras actuaciones. Las herramientas más usuales para dicho análisis son el análisis coste-beneficio y el análisis de impacto económico. Mientras que el objetivo del análisis coste-beneficio es cuantificar los beneficios y costes globales asociados a las políticas consideradas desde un punto de vista macroeconómico, el objetivo del análisis de impacto económico es cuantificar el impacto que generan unas determinadas políticas en la actividad económica de su entorno a partir de un análisis esencialmente microeconómico.

Como investigaciones previas relacionadas con la evaluación económica de las políticas de promoción de la bicicleta y sin ánimo de ser exhaustivos, podemos citar en primer lugar el análisis de la Federación Europea de Ciclistas (ECF) sobre los beneficios económicos de la bicicleta en el ámbito de la UE (ECF, 2013). Dicho estudio considera factores como los beneficios sobre la salud, la reducción de la congestión, el ahorro de combustible, la reducción en las emisiones de gases de efecto invernadero y de la contaminación de todo tipo. El resultado final arroja un beneficio neto entre 143000 y 155000 millones de euros para el conjunto de la UE-27. Otro estudio relacionado (ECF, 2014) referido a la creación de empleo asociada al uso de la bicicleta en la UE27 arroja una estimación de más de 655000 empleos para el conjunto de la UE-27 (de los cuales 23000 en España), siendo el mayor impacto en el sector del cicloturismo, con 524000 empleos en la UE-27, de los cuales 17000 en España.

En los EEUU destacamos el estudio realizado por la League of American Bicyclists en colaboración con la Alliance for Biking \& Walking (LAB-AWC 2012) que incluye análisis de diversas ciudades, entre las que destacan Portland (Oregón) y Boulder (Colorado) que mueven 
anualmente 90 y 52 millones de dólares respectivamente en negocios relacionados con la bicicleta. En Francia, un reciente estudio de L'Agence de Développement Touristique de la France (ATOUT-France, 2009) evalúa los beneficios para la salud en 15000 millones de euros y el impacto económico del cicloturismo en 1900 millones, siendo 20000 los empleos generados en dicho sector únicamente. Por su parte, la Organización Mundial de la Salud ha evaluado en un reciente estudio la potencialidad de creación de empleos en el sector de la movilidad en bicicleta, en el marco del sector más general del transporte sostenible y saludable (WHO-Europe, 2014). Los resultados obtenidos a partir de estudios específicos y del análisis de la bibliografía existente, arrojan unos resultados sin duda esperanzadores. Por ejemplo, 1,1 millones de empleos son generados actualmente en los EEUU y 278000 en Alemania.

En España, Ferri y López Quero (2011) evalúan los empleos generados en el sector de la bicicleta en algo más de 11000 en 2008, con un escenario tendencial de más de 20000 empleos para 2020 y una potencialidad, en caso de que se llevaran a cabo políticas de promoción eficientes de 78000 puestos de trabajo para ese mismo año. Otra fuente de datos a tener en cuenta en nuestro país son los informes que publica anualmente la Asociación de Marcas y Bicicletas de España sobre la economía del sector. En su informe correspondiente a 2018 (AMBE, 2019), se reporta una facturación anual de algo más de 1700 millones de euros y un total de 22000 empleos en la industria de la bicicleta de España, de los cuales 17000 lo serían en el sector del comercio minorista, cifras que están en consonancia con las ya citadas de Ferri y López Quero.

En Sevilla, el grupo de investigación liderado por el Prof. CastilloManzano de la Facultad de Económicas de la Universidad de Sevilla ha llevado a cabo un análisis coste-beneficio de la red de vías ciclistas de Sevilla (Brey et al., 2017) reportando un beneficio anual neto, tras la primera inversión inicial (estimada en torno a los 30 millones de euros), superior a los 40 millones de euros anuales, lo que resulta en un valor añadido neto y una tasa interna de retorno de 500 millones de euros y del 130\% respectivamente, estimados para el periodo 20062032. Resultados asimismo positivos del análisis coste-beneficio fueron publicados con anterioridad por la Agencia d'Ecología Urbana de Barcelona (2013), en concreto unos beneficios netos anuales también en torno a los 40 millones de euros tras la primera inversión inicial, así como una ratio beneficio-coste de 4,9, un valor añadido neto de $48 \mathrm{mi}$ llones de euros y una tasa interna de retorno del $102,5 \%$ para el periodo 2007-2013.

En lo que respecta al impacto económico, el grupo del Profesor Castillo-Manzano realizó un análisis de dicho impacto sobre la pequeña empresa local para el año 2012 (Castillo-Manzano et al., 2013) que arrojó unas ventas totales del sector de 5927 millones de euros con la creación directa de 98 empleos, así como un efecto total (directo más inducido) de 14974 millones de euros y 180 empleos. Ha transcurrido, no obstante, tiempo suficiente para que una revisión de estos resultados esté justificada, lo que constituye uno de los objetivos de este trabajo.

Como ya hemos mencionado, uno de los sectores donde mayor es el impacto económico de las políticas de promoción de la bicicleta es el sector turístico, que acapara la mayor parte del impacto económico de dichas políticas en la UE. El caso de Sevilla, como veremos a lo largo de 
este trabajo, no es una excepción, por lo que, siendo además el turismo una de las actividades económicas más importantes de la ciudad, estaría justificada una aproximación al impacto de la bicicleta en la imagen turística de la misma. Esta hipótesis se ve además justificada por la creciente presencia de la bicicleta en los reportajes de las principales agencias turísticas internacionales que resaltan la conveniencia de la bicicleta como vehículo de uso turístico para visitar la ciudad (Lonely Planet, 2012) o incluyen a Sevilla en el top ten de las mejores ciudades para visitar en bicicleta (Reuters, 2012). Es evidente que esta contribución, si bien difícil de determinar con exactitud en términos económicos, merece un primer análisis cuantitativo a través de las opiniones de los usuarios de la oferta cicloturística de la ciudad.

\section{Objetivos y metodología}

El principal objetivo de este trabajo es contribuir al análisis del impacto económico de las políticas de promoción de la bicicleta en las ciudades a través de la evaluación cuantitativa de su impacto en la pequeña empresa local y de la evolución de dicho impacto tras la implantación de la red de vías ciclistas en la ciudad de Sevilla, actualizando y ampliando el trabajo previo de Castillo-Manzano et al. (2013). Asimismo, pretendemos llevar a cabo una primera aproximación cuantitativa al análisis del impacto de dichas políticas en la imagen turística de la ciudad, a través del estudio de las percepciones de los turistas que usan la bicicleta como vehículo para visitarla, explorando así las posibilidades de mejorar la sostenibilidad del modelo turístico y de generar empleo local a través de la promoción de la bicicleta como vehículo para visitar la ciudad, ya que como veremos una parte muy importante de las empresas estudiadas se dedican a promover y organizar dicho uso. Para ello hemos desarrollado una metodología que exponemos a continuación y que esperamos pueda ser útil también para el análisis de otras ciudades que hayan sido sedes de experiencias similares, afortunadamente cada vez más comunes en nuestro país.

El análisis excluye algunas actividades económicas relacionadas con el ciclismo urbano, como por ejemplo la ejecución de nuevas infraestructuras y su mantenimiento, lo que viene justificado por el hecho de que la cuantía anual de dichas inversiones ha sido muy inferior a la del periodo inicial desde 2011 (Brey et al., 2017). También excluye la venta de bicicletas en grandes superficies y tiendas no especializadas, que según el último informe de la Asociación de Marcas y Bicicletas de España (AMBE, 2019) suponen algo más del 19\% de la facturación total del sector en España. Asimismo, se excluye del análisis el alquiler de bicicletas en hoteles y agencias no especializadas (que casi siempre, sin embargo, se realiza mediante subcontratas a empresas locales), así como los alquileres del sistema municipal de alquiler de bicicletas SEVici, por la dificultad de analizarlo separadamente de otras actividades de la empresa matriz, de ámbito nacional y actividades mucho más diversificadas.

También se han excluido del análisis las actividades económicas relacionada con la creciente presencia en las vías ciclistas de la ciudad de patinetes eléctricos, que representan en la actualidad algo más del 10\% del tráfico en las vías ciclistas (Hernández-Herrador y Marqués, 2019), debido sobre todo a la dificultad de cuantificar las actividades de este sector, por su novedad. En todo caso, muchas de las empresas que se dedican a esta actividad se dedican también a actividades relacionadas 
con la bicicleta, por lo que es posible que en el futuro ambos sectores converjan en uno solo.

En todo caso, consideramos que el análisis realizado captura los aspectos más relevantes del impacto del ciclismo urbano en la economía local de la ciudad, dejando aparte solo contribuciones marginales, que en todo caso se podrían estimar a partir de los resultados de este estudio o ser objeto de un estudio posterior.

La metodología utilizada se basa en un trabajo de campo realizado fundamentalmente durante los meses de otoño de 2019 y estructurado en tres fases:

- Identificación de las empresas del sector, casi siempre a partir de su presencia en redes sociales.

- Obtención de datos mediante encuestas on-line, investigación de los datos del Boletín del Registro Mercantil (BORME) y entrevistas presenciales.

- Análisis de los datos obtenidos.

Una de los problemas metodológicos más recurrentes ha sido la dificultad para establecer la pertenencia o no al sector de empresas dedicadas simultáneamente a la venta de bicicletas y motocicletas y sus accesorios. Para ello hemos recurrido al análisis cualitativo de su presencia en redes sociales (páginas Web y perfiles de Facebook) así como a la inspección directa. En el caso de los datos de facturación hemos recurrido, siempre que ha sido posible, a los últimos datos disponibles del BORME. Sin embargo, en muchos casos no ha sido posible obtener dichos datos, por lo que hemos tenido que recurrir a la mencionada encuesta y a entrevistas y/o inspección directa. Para la evaluación del empleo creado hemos confiado en primer lugar en las encuestas y en segundo en los datos del BORME, que en general mostraron pequeñas diferencias cuando fue posible obtenerlos.

El ámbito geográfico del estudio ha sido la ciudad de Sevilla, por lo que se han seleccionado empresas que, o bien tenían su sede social en dicho municipio, o bien realizaban su actividad comercial fundamentalmente en dicha ciudad, teniendo su sede en el entorno inmediato de la misma.

En la citada encuesta se preguntaba por las actividades de la empresa, lo que nos ha permitido dividir el sector, para su análisis, en tres categorías:

- Categoría 1: Empresas que podríamos calificar de "tradicionales" dedicadas fundamentalmente a la venta presencial de bicicletas y accesorios o a su reparación, incluyendo bicicletas eléctricas.

- Categoría 2: Empresas dedicadas fundamentalmente al alquiler y a la organización de rutas en bicicleta, casi siempre como actividad turística, pero también a veces como actividad formativa.

- Categoría 3: Empresas que podríamos calificar como de nuevos servicios, lo que incluye actividades como venta on-line, fabricación de ciclos para actividades específicas, transporte de mercancías (ciclologística), gestión de aparcamientos de bicicletas, publicidad en bici, consultoría y actividades de promoción de la bicicleta (cursos, plataformas de promoción, etc.).

El estudio cuantitativo se ha completado con el análisis de las percepciones de los empresarios del sector acerca de cómo ha influido la 
creación de la red de vías ciclistas de la ciudad en el desarrollo de sus actividades económicas y de las acciones que ellos propondrían para mejorarla.

Como ya hemos mencionado en la Introducción, el sector del cicloturismo es uno de los subsectores más importantes, en cuanto a facturación y empleo, del sector del ciclismo en el conjunto de la UE. Como se verá a continuación también lo es en Sevilla, por lo que, siendo el turismo además un sector económico de vital importancia en la ciudad, nos ha parecido de interés hacer una evaluación del impacto del cicloturismo urbano en la imagen turística de la ciudad y de su potencial para avanzar hacia un modelo de turismo más sostenible. Para ello se pidió a una serie de empresas representativas de la Categoría 2 mencionada (alquiler y rutas turísticas en bicicleta) que pasaran a sus clientes un cuestionario donde se les preguntaba por su grado de conocimiento previo de la infraestructura ciclista de la ciudad y por su valoración de la misma. El análisis de las respuestas a dicha encuesta se ofrece en el apartado de resultados de este trabajo.

\section{Resultados}

\section{Caracterización del sector}

Tras el trabajo de campo realizado y tras eliminar duplicaciones y empresas que no satisfacían las condiciones establecidas en el apartado anterior, fueron identificadas un total de 56 empresas con una facturación estimada superior a los 9 millones de euros que daban empleo a 200 personas en total. Los datos agregados correspondientes a dichas empresas se muestran en la primera fila de la Tabla 1. El número de empleos por empresa se sitúa entre tres y cuatro, lo que indica que el sector está dominado por la pequeña empresa, mientras que las ventas por empleado se sitúan en torno a los 48000 euros, un dato bastante inferior a la media española para la pequeña empresa, que se sitúa en torno a los 200000 euros por empleado (Círculo de Empresarios, n.d.), lo que evidencia que estamos ante un sector intensivo en mano de obra, es decir con una alta capacidad de creación de empleo por euro facturado.

A efectos de comparación también se han incluido en la tabla los datos del análisis de Castillo-Manzano et al. (2013) para el año 2012. Como se puede observar el sector ha experimentado un notable incremento desde esa fecha, del $62 \%$ en facturación y del $104 \%$ en empleo. Las ventas por empleado han disminuido, lo que se ha traducido en un mayor número de trabajadores por empresa. Finalmente, las últimas columnas de la tabla incluyen los datos del impacto total (directo más indirecto) de las actividades económicas consideradas en la economía local. Los datos para 2019 han sido calculados utilizando los mismos multiplicadores obtenidos en Castillo-Manzano et al. (2013), por lo que deben tomarse con cautela, pues son estimaciones.

Tabla 1. Principales datos agregados del sector para los años 2019 y $\left.2012 .\left.\right|^{*}\right)$ Estimaciones (ver texto). Fuente: Elaboración propia.

\begin{tabular}{|l|l|c|c|c|c|c|c|}
\hline Año & $\begin{array}{l}\text { Número de } \\
\text { empresas }\end{array}$ & $\begin{array}{l}\text { Facturación } \\
\text { (miles de euros) }\end{array}$ & Empleos & $\begin{array}{l}\text { Facturación } \\
\text { por empleo }\end{array}$ & $\begin{array}{l}\text { Empleos por } \\
\text { empresa }\end{array}$ & $\begin{array}{l}\text { Impacto eco- } \\
\text { nómico (mi- } \\
\text { les de euros) }\end{array}$ & \begin{tabular}{c} 
Empleo total \\
\hline 2019
\end{tabular} \\
\hline 2612 & 42 & 9592 & 200 & $48000 €$ & 3,57 & $24233(*)$ & $367(*)$ \\
\hline
\end{tabular}


La Tabla 2 muestra los datos de la Tabla 1 desagregados según las categorías definidas en el apartado anterior. Puede observarse como los subsectores ligados al alquiler y a los nuevos modelos de negocio (Categorías 2 y 3) presentan, como era de esperar, una ratio de ventas por empleado inferior y un número de empleados por empresa superior a las cifras correspondientes al subsector de venta de bicicletas (Categoría 1). En cuanto al sector en su conjunto, presenta una composición bastante equilibrada entre las empresas más tradicionales de venta presencial de bicicletas (Categoría 1) y las empresas de servicios y nuevos modelos de negocio (Categorías 2 y 3 ).

\begin{tabular}{|l|c|c|c|c|c|}
\hline & $\begin{array}{l}\text { Número de } \\
\text { empresas }\end{array}$ & $\begin{array}{l}\text { Facturación } \\
\text { (miles de euros) }\end{array}$ & Empleos & $\begin{array}{l}\text { Facturación por } \\
\text { empleo }\end{array}$ & $\begin{array}{l}\text { Empleos por } \\
\text { empresa }\end{array}$ \\
\hline Categoría 1 & 27 & 4913 & 48 & 102345 & 1,78 \\
\hline Categoría 2 & 20 & 2996 & 108 & 27737 & 5,40 \\
\hline Categoría 3 & 9 & 1684 & 44 & 38267 & 4,89 \\
\hline Total & 56 & 9592 & 200 & 47960 & 3,57 \\
\hline
\end{tabular}

En la Figura 1 hemos representado el número total de empresas clasificadas por categorías y por año de constitución: anterior o posterior a la fecha de inicio de las políticas de promoción que dieron lugar a la actual red de vías ciclistas de la ciudad. Es interesante observar como la mayoría de las empresas de las categorías 2 y 3 fueron creadas con posterioridad a dicha fecha, si bien gran parte de las empresas de la categoría 1 también fueron asimismo creadas tras dicha fecha. Puede concluirse por tanto que, si bien la creación de la red de vías ciclistas tuvo un impacto considerable en la economía local de la bicicleta, este impacto fue todavía mayor en los subsectores del alquiler turístico de bicicletas (categoría 2) y de los nuevos modelos de negocio (categoría 3). Esta evolución explica además el mayor incremento en la creación de empleo en comparación con el incremento de la facturación mostrada en la Figura 1, que sería debida a este mayor crecimiento relativo de los subsectores ligados al alquiler turístico y a los nuevos modelos de negocio, más intensivos en fuerza de trabajo.

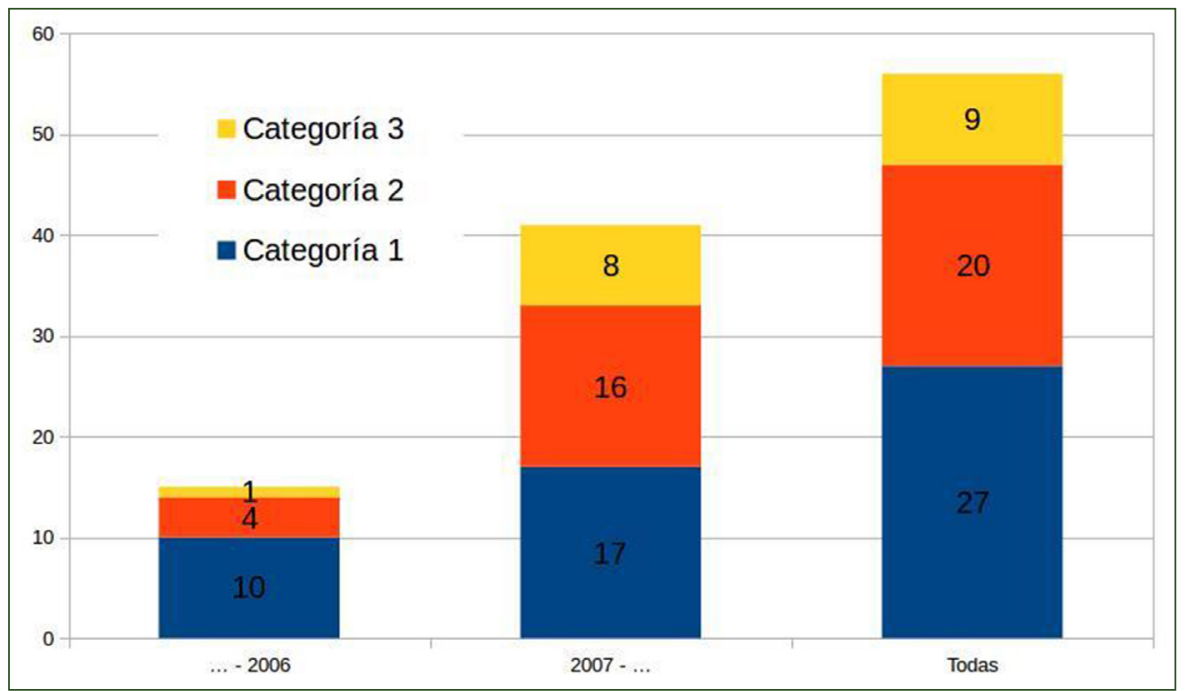

Tabla 2. Principales datos del sector desagregados por categorías (año 2019). Fuente: Elaboración propia.

Figura 1. Año de constitución de las empresas analizadas por categorías. Fuente: Elaboración propia. 


\section{Percepciones de las empresas}

Como ya se ha mencionado se elaboró un cuestionario que fue completado on-line o mediante entrevistas presenciales con el objetivo tanto de complementar los datos obtenidos del BORME como de conocer las percepciones de las empresas en relación con el impacto de la creación de la red de vías ciclistas de la ciudad. Un total de 47 empresas respondieron el cuestionario. A la pregunta genérica de si consideraban que ello había influido de manera sustancial en las empresas del sector, la inmensa mayoría de las empresas (y la totalidad de las empresas de la categoría 2) contestaron que "mucho". Ninguna empresa valoró que dicho influjo había sido nulo. La Figura 2 muestra las respuestas, ordenadas por categorías, a la pregunta más específica de cómo había influido la creación de la red de vías ciclistas en su empresa en particular. La mayoría de las empresas consideran que dicha influencia había sido grande, aunque algunas empresas consideran sin embargo que tal influjo ha sido pequeño o inexistente. El análisis pormenorizado de éstas últimas respuestas lleva a la conclusión de que se trata en su mayoría de empresas que, por su actividad, no están relacionadas con el ciclismo urbano, casi siempre por dedicarse al cicloturismo deportivo o de naturaleza, por lo que la conclusión obvia parece ser que la creación de la red de vías ciclistas ha tenido poca o nula relación con los negocios no directamente relacionados con el ciclismo urbano.

Por categorías, son las empresas dedicadas al alquiler y la organización de rutas turísticas las que más valoran el impacto de la red de vías ciclistas sobre sus negocios, con la única excepción de las ya mencionadas que se dedican a rutas de naturaleza. Les siguen las empresas tradicionales de venta y reparación de bicicletas, que también reconocen en general un gran impacto de la creación de la red en sus cifras de negocio, aunque en menor medida. Paradójicamente son las empresas dedicadas a nuevas actividades, surgidas en su mayoría tras la creación de la red, las que menos la valoran, probablemente por ese mismo motivo.

También se preguntó por las mejoras a realizar en la red de vías ciclistas. En concreto se preguntó por la importancia de mejoras tales como la ampliación o el mejor mantenimiento de la red, la señalización turística de la misma y la creación de más plazas de aparcamiento de bicicletas a lo largo de la red. Un $90 \%$ de los encuestados se declaró a favor de un mejor mantenimiento, lo que parece indicar una cierta preocupación por el deterioro de las vías ciclistas tras más de 10 años

Figura 2. Percepción de las empresas acerca de la influencia de la red de vías ciclistas en su negocio en particular, ordenadas por categorías. Fuente: elaboración propia.

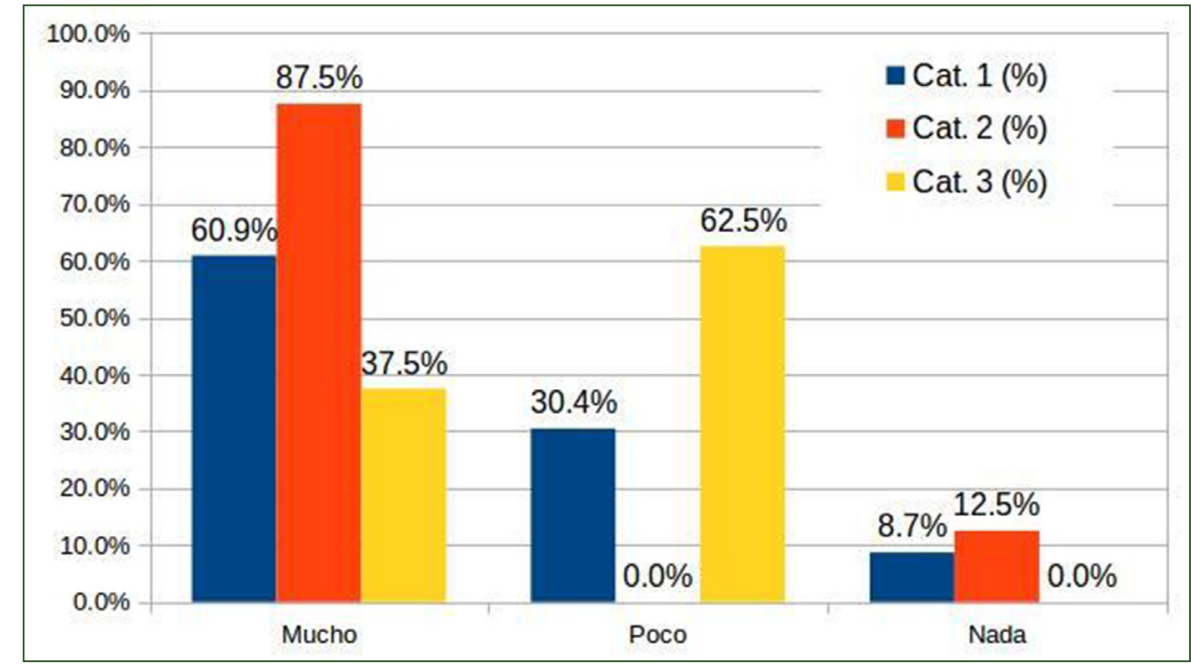


de funcionamiento. Le siguieron, por orden de importancia, las demandas de ampliación de la red (81\%), de más aparcamientos $(77 \%)$ y de una mejor señalización turística $(73 \%)$.

Finalmente, y aunque ello no se contemplaba explícitamente en el cuestionario, durante las entrevistas mantenidas con empresarios del sector pudimos detectar una creciente preocupación relacionada con el "intrusismo" y/o "competencia desleal" por parte de empresas de alquiler de bicicletas y patinetes eléctricos que operan en la ciudad sin locales fijos y aparentemente sin licencia municipal.

\section{Percepciones de los usuarios de los servicios de alquiler}

Como ya hemos visto (Figura 1) son los negocios relacionados con el alquiler de bicicletas, la mayoría de ellos orientados al turismo, los que más han crecido desde la creación de la red de vías ciclistas de la ciudad. En la actualidad, la facturación total de este sector alcanza los 3 millones de euros al año (ver Tabla 2), lo que a un coste estimado de $8 €$ por alquiler ${ }^{4}$ supondría unos 375000 alquileres al año, la mayoría de los cuales se utilizan para visitar la ciudad y, por tanto, hacen uso de la red de vías ciclistas de la misma. A estos alquileres habría que añadir los más de 8000 abonos de corta duración ${ }^{5}$ del servicio municipal de alquiler de bicicletas SEVici (JCDecaux, 2018) y, más recientemente, los servicios de alquiler de patinetes y bicicletas eléctricas sin estación, cuyo uso por los turistas es difícil de cuantificar. Se trata pues de unos servicios ampliamente utilizados, con un impacto sin duda importante en la imagen de la ciudad y directamente derivados de la existencia de una red de vías ciclistas.

A fin de evaluar dicho impacto se realizaron un total de 482 encuestas a los clientes de un total de 12 empresas de alquiler de bicicletas que accedieron a colaborar en el estudio. La encuesta se presentaba como un "encuesta de calidad" acerca de la red de vías ciclistas y se preguntaba por: 1) El conocimiento previo sobre la existencia de dicha red antes del viaje; 2) En qué medida influyó la existencia de dicha red en su decisión de visitar Sevilla; 3) Su valoración global de dicha red; 4) Hasta qué punto consideraban a Sevilla una ciudad "amable" para los y las ciclistas; y 5) En qué medida influirá su conocimiento de la red de vías ciclistas a la hora de recomendar visitar Sevilla a sus amigos y conocidos. Las valoraciones se realizaban todas de 0 a 3 , entre 0 (nada) y 3 (mucho). Finalmente se preguntaba por algunos datos básicos, como edad, género, o país de origen.

Respondieron a la encuesta personas de 44 países diferentes, incluida España, que fueron clasificadas en dos categorías: países de tradición ciclista ${ }^{6}$ y el resto. Del total de las personas que respondieron a la encuesta, la mitad (50\%) fueron mujeres y algo menos de la mitad (46\%) hombres, mientras que un $4 \%$ dejaron esta casilla en blanco. En cuanto a la edad, las respuestas se repartieron bastante homogéneamente entre los 20 y los 50 años, descendiendo algo el porcentaje de encuestados por arriba de 50 años y por debajo de 20, pero siendo su presencia todavía bastante significativa.

Una primera conclusión relevante de la encuesta es que la mayoría de los encuestados (59\%) decía desconocer la existencia de la red de vías ciclistas de Sevilla con anterioridad a su visita, lo que parece indicar un bajo conocimiento a nivel internacional (la mayoría de las respuestas negativas se concentraron en visitantes extranjeros) de los esfuerzos realizados por la ciudad para mejorar su ciclabilidad, pese a los reconocimientos que dichos esfuerzos han alcanzado por parte de di-
4 Estimación realizada sobre la base de las opiniones de los empresarios del sector. $5 \quad$ El servicio municipal de alquileres SEVici ofrece la posibilidad de obtener un abono semanal por 13,33 euros, pensado fundamentalmente para ser usado por los visitantes de la ciudad.

6 Países europeos que superan el 10\% de desplazamientos en bicicleta según el Eurobarómetro especial 422a "Quality of Transport” de la Comisión Europea (European Commission, 2014). Estos países son: Alemania, Bélgica, Dinamarca, Finlandia, Hungría, Noruega, Países Bajos y Suiza. 
Figura 3. Valoraciones de los clientes de las empresas de alquiler sobre las preguntas A), B), C) y D) indicadas en el texto. Fuente: Elaboración propia. versos organismos internacionales (Marqués et al., 2015). En la Figura 3 se representan las diversas valoraciones alcanzadas por la red de vías ciclistas entre los encuestados:

a) ¿En qué medida influyó la existencia de la red de vías ciclistas al decidir su visita a Sevilla? (solo para los que ya conocían previamente la existencia de la red de vías ciclistas).

b) ¿Podría valorar la red de vías ciclistas?

c) ¿Hasta qué punto piensa que Sevilla es una ciudad amable para los/las ciclistas?

d) ¿En qué medida influirá la existencia de la red de vías ciclistas al recomendar Sevilla a sus amigos o familiares?

Los datos, resumidos en las Figura 3, indican una gran importancia de la existencia de la red de vías ciclistas en la decisión de visitar Sevilla (para aquellos turistas que ya conocían este hecho). Un $76 \%$ de los encuestados reconocen alguna influencia de este hecho en su decisión y un $32 \%$ afirman que les ha influenciado "mucho". La valoración de la red ciclista es muy alta, así como la de la ciclabilidad de la ciudad y resulta significativo el alto porcentaje de visitantes que afirman que la existencia de la red de vías ciclistas es un factor importante a la hora de recomendar Sevilla como destino turístico a sus amigos y familiares. Esta valoración positiva de la red de carriles-bici de la ciudad es bastante homogénea entre toda la población encuestada, aunque ligeramente más alta entre la población extranjera que entre los nacionales. Asimismo, los visitantes de países con menor tradición ciclista valoran la red ligeramente mejor que los visitantes de países con mayor tradición ciclista, algo que cabe atribuir que éstos últimos están acostumbrados a estándares de calidad más altos. Las mujeres valoran la red ciclista, en general, mejor que los hombres, lo que parece indicar una mayor valoración general por parte de las mujeres de este tipo de infraestructuras, algo que otros estudios han puesto ya de manifiesto (Garrard, Handy y Dill, 2012).

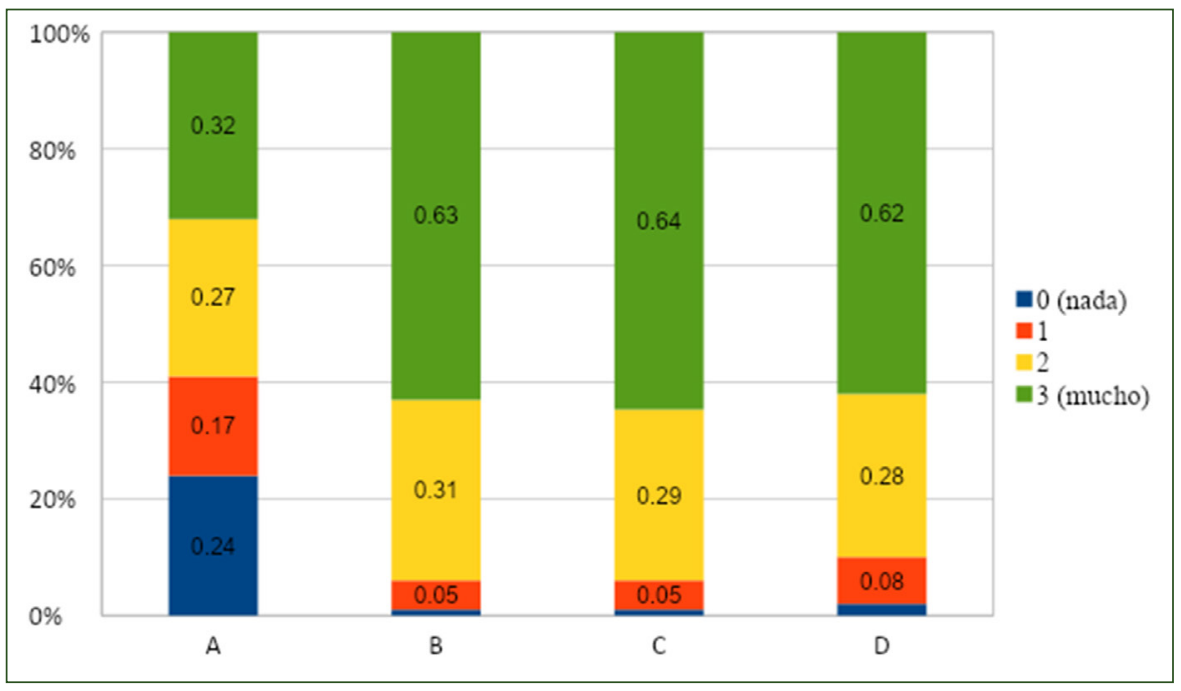

\section{Conclusiones y recomendaciones}

Se ha desarrollado una metodología para el estudio del impacto de la creación de una red de vías ciclistas segregadas del tráfico motorizado en la economía local y en la imagen turística de las ciudades, que se ha 
aplicado al análisis del caso concreto de la ciudad de Sevilla. Pensamos que esta metodología podría también ser de utilidad en el análisis de procesos similares en otras ciudades.

El estudio realizado muestra como la creación de una red de vías ciclistas, segregadas del tráfico motorizado, ha dinamizado el sector económico de la bicicleta en Sevilla, un sector en franco crecimiento tras la creación de dicha red, tanto en lo que se refiere a la facturación global (más de 9 millones de euros) como a la creación de empleo (200 empleos directos y una estimación de más de 350 empleos totales, directos más indirectos) lo que contrasta con la evolución general de la economía local, en franco descenso desde 2007 (Ayuntamiento de Sevilla, 2018; Ayuntamiento de Sevilla, 2019). Este crecimiento ha sido importante, tanto en el número de empresas como en la facturación y en el empleo. En lo que respecta a la facturación, el sector más tradicional de la venta y reparación continúa siendo líder, mientras que, en lo que respecta a la creación de empleo, ésta se ha concentrado sobre todo en el subsector del alquiler de bicicletas y oferta de rutas turísticas en bicicleta, probablemente como consecuencia de la mayor necesidad de trabajadores (guías, monitores, etc.) en este sector. Este subsector del alquiler y rutas turísticas ha sido también el que ha experimentado un mayor crecimiento en el número de empresas desde 2007. Resulta también significativa la aparición de un tercer sector de empresas a partir de 2007, dedicadas a nuevos servicios como venta on-line, consultoría y promoción social de la bicicleta, ciclologística, fabricación de bicicletas (triciclos), gestión de aparcamientos de bicicletas y publicidad en bicicleta, lo que demuestra el dinamismo del sector.

Los empresarios son conscientes de la importancia de la creación de la red de vías ciclistas para su negocio. La mayoría de ellos califican este impacto como muy alto, siendo esta calificación más alta entre los empresarios del subsector del alquiler y rutas turísticas. Sin embargo, también muestran su inquietud ante ciertas carencias de la red, especialmente en lo que respecta al creciente deterioro de la misma, siendo casi unánime $(90 \%)$ la demanda de un mejor mantenimiento, por lo que sería necesario atender esta demanda, que también ha sido formulada en reiteradas ocasiones por los usuarios en general y medios de comunicación locales.

El impacto positivo de la red de vías ciclistas en la imagen turística de la ciudad es evidente, como lo ponen de manifiesto las referencias a la misma en las más importantes guías turísticas a nivel internacional, algunas de ellas citadas en la Introducción. El análisis de las percepciones de los visitantes que hacen uso de esta infraestructura demuestra que, en general, la valoran muy positivamente y que consideran que su existencia es un buen argumento a la hora de decidir/recomendar una visita turística a Sevilla. No obstante, resulta llamativo el alto grado de desconocimiento previo que la mayoría de los visitantes muestran con respecto a su existencia, que en cambio valoran luego muy positivamente, por lo que serían necesarias acciones encaminadas a promover un mayor conocimiento a nivel tanto nacional como internacional de dicha infraestructura en congresos, ferias, encuentros de empresas y operadores de turismo, etc., tanto específicos como de carácter general, dado que todo parece indicar que dichas actuaciones podrían tener un impacto muy positivo en la promoción de la ciudad como destino turístico sostenible.

Aparte de las actuaciones ya mencionadas de mejor mantenimiento y promoción del conocimiento de la red fuera de Sevilla, dada la cons- 
tatación de que nos hallamos ante un sector especialmente dinámico de la economía local, serían necesarias acciones encaminadas a su protección y promoción, evitando que se genere competencia desleal por parte de grandes empresas que puedan llegar a competir sobre la base de un incumplimiento sistemático de las normas vigentes en lo referente a la ocupación de la vía pública o el ejercicio en la misma de actividades económicas sin la preceptiva autorización, algo que, como hemos podido detectar, preocupa a los empresarios del sector y, en especial, a las empresas de alquiler de bicicletas y organización de rutas turísticas. Asimismo, la previsible creación de "zonas de bajas emisiones" en los centros urbanos, tras la inclusión de esta figura en el Borrador del Plan Nacional Integrado de Energía y Clima (PNIEC) (Ministerio de para la Transición Ecológica y el Reto Demográfico, 2020) abre posibilidades interesantes para el fomento de la ciclologística, del que ya existen empresas e iniciativas en el sector, aunque todavía incipientes.

\section{Nota final}

Este trabajo ha sido elaborado sobre la base de un trabajo de campo realizado con anterioridad al estado de alarma decretado como consecuencia de la COVID-19. En consecuencia, no hemos podido incluir en el análisis el impacto de dicha pandemia en el sector. 


\section{Referencias bibliográficas}

Agencia d'Ecología Urbana de Barcelona (2013). Análisis Socioeconómico de la Red de Carriles Bici de la Ciudad de Sevilla. Sevilla: Junta de Andalucía.

AMBE (2018). El sector de la Bicicleta en Cifras - 2018. Recuperado el 25 de febrero de 2020 de: http:/ / asociacionambe.es/wp-content/uploads/2019/06/ Presentaci\%C3\%B3n-Nota-de-Prensa-2018_AMBE. pdf.

ATOUT-France (2009). Spécial économie du vélo. Recuperado el 23 de febrero de2020 de: http:/ / www. atout-france.fr/system/files/73/2014/10/2009-pdfsom-economie-velo.pdf.

Ayuntamiento de Sevilla (2007). Plan de la Bicicleta de Sevilla 2007-2010. Recuperado el 23 de febrero de 2020 de: https://www.urbanismosevilla.org/areas / sostenibilidad-innovacion/sevilla-en-bici/ficheros/ pdfs / plan-director-de-la-bicicleta-2007-2010/.

Ayuntamiento de Sevilla (2014). Programa de la Bicicleta 2020. Recuperado el 23 de febrero de 2020 de: https://www.urbanismosevilla.org/areas/sostenibilidad-innovacion/sevilla-en-bici/ficheros/pdfs/programa-de-la-bicicleta-sevilla-2020/ programa-de-labicicleta-sevilla-2020/at_download/file.

Ayuntamiento de Sevilla (2018) Anuario Estadístico Ciudad de Sevilla 2017. Recuperado el 23 de febrero de 2020 de: https://www.sevilla.org/servicios/serviciode-estadistica/datos-estadisticos/anuarios/2017/ publicacion/anuario-estadistico-2017.pdf.

Ayuntamiento de Sevilla (2019). Plan de Movilidad Urbana Sostenible del Municipio de Sevilla. Diagnóstico. Recuperado el 25 de febrero de 2020 de: https: / / www.sevilla.org/actualidad/blog/plan-de-movilidad-urbana-sostenible-de-sevilla/pmus-sevilla-diagnostico_v34.pdf.

Brey, Raul; Castillo-Manzano, José I.; Castro-Nuño, Mercedes; López-Valpuesta, Lourdes; MarchenaGómez, Manuela; y Sánchez-Braza, Antonio (2017). Is the widespread use of urban land for cycling promotion policies cost effective? A Cost-Benefit Analysis of the case of Seville. Land use policy, 63, 130-139. DOI: 10.1016/j.landusepol.2017.01.007.

Castillo-Manzano José Ignacio et al. (2013). Guía para la Medición del Impacto Económico y Social de la Política de promoción de las Bicicletas: Aplicación a Sevilla. Recuperado el 25 de febrero de 2020 de: https:/ / www.aopandalucia.es/inetfiles/resultados_IDI/ GGI3001IDIR/memoria/MEMORIA_FINAL_ GGI3001IDIR.pdf.

Círculo de Empresarios (2019). La Empresa Mediana Española. Informe Anual 2018. Recuperado el 25 de febrero de 2020 de: https:/ / circulodeempresarios.org/app/uploads/2019/01/Empresa-media-
na-espa\%C3\%B1ola-informe-anual-2018-Circulode-Empresarios.pdf.

Dekoster, J. y Schollaert, U. (1999). Cycling: the way ahead for towns and cities. Luxemburg: Oficina de Publicaciones Oficiales de las Comunidades Europeas. Recuperado el 23 de febrero de 2020 de: http:/ / ec.europa.eu/environment/archives / cycling/cycling_en.pdf.

European Commission (2014). Special Eurobarometer 422 $2^{a}$ QUALITY OF TRANSPORT. Recuperado el 25 de febrero de 2020 de: https://ec.europa.eu/ commfrontoffice/publicopinion/archives/ebs/ ebs_422a_en.pdf.

ECF (2013). Calculating the economic benefits of cycling in EU-27. Recuperado el 23 de febrero de 2020 de: https:/ / ecf.com/sites/ecf.com/files/Fabians \% 20ECF_Economic-benefits-of-cycling-inEU-27-3.pdf.

ECF (2014). Cycling Works, Jobs and Job Creation in the Cycling Economy. Recuperado el 23 de febrero de 2020 de: https:/ / ecf.com/sites/ecf.com/ files / 141125-Cycling-Works-Jobs-and-Job-Creationin-the-Cycling-Economy.pdf.

Estevan, Antonio; y Sanz, Alfonso (1996). Hacia la Reconversión Ecológica del Transporte en España. Bilbao: Bakeaz.

Ferri, Manel; y López Quero, Manuel (2011). La generación de empleo en el transporte colectivo en el marco de una movilidad sostenible. Madrid: Fundación Conde del Valle de Salazar. Recuperado el 23 de febrero de 2020 de: https://www.empleaverde.es/sites/default/files/publicaciones/transportecolectivo.pdf.

Garrard, Jan; Handy, Susan; Dill, Jennifer (2012). Women and Cycling. En Pucher, John; y Buehler, Ralph (eds.) City Cycling. Cambridge, MA: MIT Press.

Hernández-Herrador, Vicente; y Marqués, Ricardo (2019). Análisis del uso de las Vías Ciclistas en la Ciudad de Sevilla. Sevilla: Acontramano. Recuperado el 25 de febrero de 2020 de: http:/ / www.acontramano.org $/$ index.php?option $=$ com_joomdoc\&view $=$ do cuments\&path=conteos-e-informes-del-uso-de-la-bici-en-sevilla/2019-analisis-del-uso-de-las-vias-ciclistascarriles-bici-en-la-ciudad-de-sevilla\&Itemid $=62$.

JCDecaux (2018). Memoria Anual SEVici 2017. Sevilla: Ayuntamiento de Sevilla-Gerencia de urbanismo y Medio Ambiente. Recuperado el 25 de febrero de 2020 de: https://www.urbanismosevilla.org/areas/ sostenibilidad-innovacion/sevilla-en-bici/ficheros/ informes-sevici/informe-anual-sevici-2017/view.

LAB-AWC (2012). Bicycling Means Business: The Economic Benefits of Bicycle Infrastructure. Recuperado el 23 de febrero de 2020 de: https:/ / www.bikeleague. 
org/sites/default/files/Bicycling_and_the_Economy-Econ_Impact_Studies_web.pdf.

Lonely Planet (2012). Sevilla goes green. Recuperado el 25 de febrero de 2020 de: https:/ / www.lonelyplanet.com/articles/seville-goes-green.

Marqués, Ricardo; Hernández-Herrador, Vicente; Calvo-Salazar, Manuel; y García-Cebrián, José Antonio (2015). How infrastructure can promote cycling in cities: Lessons from Seville, Research in Transportation Economics, 53, 31-44. DOI: 10.1016/j.retrec.2015.10.017.

Marqués, Ricardo (2017). La Importancia de la Bicicleta. Sevilla: Editorial de la Universidad de Sevilla.

Ministerio para la Transición Ecológica y el Reto Demográfico (2020). Plan Nacional Integrado de Energía y Clima (Borrador). Recuperado el 24 de febrero de 2020 de: https://www.idae.es/informacion-y-publicaciones / plan-nacional-integrado-de-energia-y-clima-pniec-2021-2030.

Reuters (2012, 11 de mayo). Top ten cycling destinations [entrada de blog]. Reuters. Recuperado el 25 de febrero de 2020 de: https:/ /www.reuters.com/ article/uk-travel-picks-cycling/top-10-cycling-destinations-idUSLNE84A01Q20120511.

Sanz, Alfonso; Pérez-Senderos, Rodrigo; y Fernández, Tomás (1996). La Bicicleta en la Ciudad. Manual de políticas y diseño para fomentar el uso de la bicicleta como medio de transporte. Madrid: Ministerio de Fomento.

Sanz, Alfonso; Vega, Pilar; y Mateos, Miguel (2014). Cuentas Ecológicas del Transporte en España. Madrid: Libros en acción. Recuperado el 24 de febrero de 2020 de: http:/ / www.ecologistasenaccion.org/ IMG/pdf/info_cuentas-ecologicas.pdf.

Sanz, Alfonso; Mateos, Miguel; Vega, Pilar; Coq Huelva, Daniel; Delgado Cabeza, Manuel; Márquez, Carolina; Pérez, David; y Rodríguez Morillas, Carmen (2015). Las Cuentas Integradas del Transporte en Andalucía. Granada: GEA 21. Recuperado el 24 de febrero de 2020 de: https:/ / archive.org/details/LasCuentasIntegradasDelTransporteEnAndalucia.

WHO-Europe (2000). Transport, Environment and Health. Recuperado el 24 de febrero de 2020 de: http:/ / www.euro.who.int/__data/assets/pdf_ file $/ 0003 / 87573 /$ E72015.pdf?ua $=1$.

WHO-Europe (2014). Unlocking new opportunities. Jobs in green and healthy transport. Recuperado el 23 de febrero de 2020 de: https:/ / thepep.unece.org/sites/default/files/2017-05/Unlocking-new-opportunities-jobs-in-green-and-health-transport-Eng.pdf.

Marqués, Ricardo; López-Peña, Manuel; y Hernández-Herrador, Vicente (2020). Contribución al análisis del impacto de la creación de una red de vías ciclistas en la economía local y en la imagen turística de las ciudades. El caso de Sevilla. Hábitat y Sociedad, 13, 193-206.

<http://dx.doi.org/10.12795/HabitatySociedad.2020.i13.11>

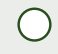

